\title{
Increased Sample Yield and Achievable Imaging Resolutions Through Thin Film Transfer Technique
}

\author{
Otto Zietz ${ }^{1}$, Sam Olson ${ }^{1}$, Brendan Coyne ${ }^{1}$ and Jun Jiao ${ }^{1}$ \\ ${ }^{1 .}$ Department of Mechanical and Materials Engineering, Portland State University, Portland, OR.
}

Metal thin film catalyzed synthesis of graphene continues to be an area of intense research. However, characterization of catalyst morphology effects on resultant graphene quality is increasingly difficult due to grainsize reduction as synthesis temperatures and catalyst thickness are decreased [1]. An electrochemical transfer method for metal thin films was developed, enabling millimeter sized portions of the catalyst film to be examined via transmission electron microscopy techniques. This technique significantly simplifies the sample preparation processes compared to traditional TEM sample preparation for planar view characterization.

Electrochemical transfer of graphene from metal foils and thin films with strong adhesion to the supporting substrate have been reported [2]. Our study suggests that thin films with weak adhesion to $\mathrm{SiO}_{2}$ would delaminate during the graphene transfer process. This phenomenon was then optimized to enable large scale transfer of metal thin film catalysts from $\mathrm{SiO}_{2}$. The thin film, a $50 \mathrm{~nm} \mathrm{NiCu}$ alloy supported by $\mathrm{SiO}_{2}$, is placed in a minimally reactive electrolyte, $\mathrm{NaOH}$, and acts as an anode to a platinum cathode (Fig 1a). Following initial delamination of the edge of the film, it is lowered into the electrolyte at an angle such that $\mathrm{O}_{2}$ bubble generation occurs primarily at the $\mathrm{SiO}_{2} /$ metal interface. The floating film is then washed and transferred to a TEM grid (Fig 1c) and imaged in an SEM Transmission Kikuchi Diffraction (TKD) holder (Fig 1b).

A TKD Inverse Pole Figure (IPF) Z map of the transferred thin film (Fig 2a) was compared to an Electron Backscatter Diffraction (EBSD) IPF Z map (Fig 2d) of the catalyst on $\mathrm{SiO}_{2}$ support to demonstrate the order of magnitude improvement in spatial resolution achieved with the TKD process. Maps were taken with $10 \mathrm{~nm}$ step sizes on catalyst thin films from a single CVD graphene synthesis. Maps and Forward Scatter Detector (FSD) images were taken with the transferred film at $-20^{\circ}$ tilt with respect to the incident beam (Fig 2c) and the as-synthesized film at $70^{\circ}$ tilt (Fig 2d). The increase in achievable spatial resolution is most prominently displayed in the presence of twinning and clear grain boundaries in the TKD map, while similar locations in the EBSD map are often unresolved. This improvement is associated with increased Kikuchi pattern strength (Fig 2b,f) resulting from a reduced interaction volume in the transferred film compared to the $\mathrm{SiO}_{2}$-supported film and the reduced sensitivity to surface morphology during TKD acquisition.

TEM inspection of the transferred catalyst revealed a suspended non-metal thin film near edges of a hole in the catalyst (Fig 3a). Much of the suspended film was damaged and curled (Fig 3b); however, examining near the interface and focusing on the plane of the metal enabled simultaneous observation of lattice fringes for both the catalyst and the non-metal film (Fig 3c). Measurements and FFT of these regions of interest reveal characteristic lattice spacing of Ni (Fig 3d) and graphene (Fig 3e), indicating that the electrochemical transfer method is primarily active at the $\mathrm{SiO}_{2} /$ metal interface, leaving the graphene on the top surface largely intact [3].

References: 
[1] J Cho, M Cullinan, ASME J. Micro Nano-Manuf 6 (2017), p. 024501.

[2] Y Wang, Y Zheng, X Xu, ACS Nano 5 (2011), p. 9927.

[3] The authors would like to thank Intel, NSF, and the Oregon Metals Initiative.
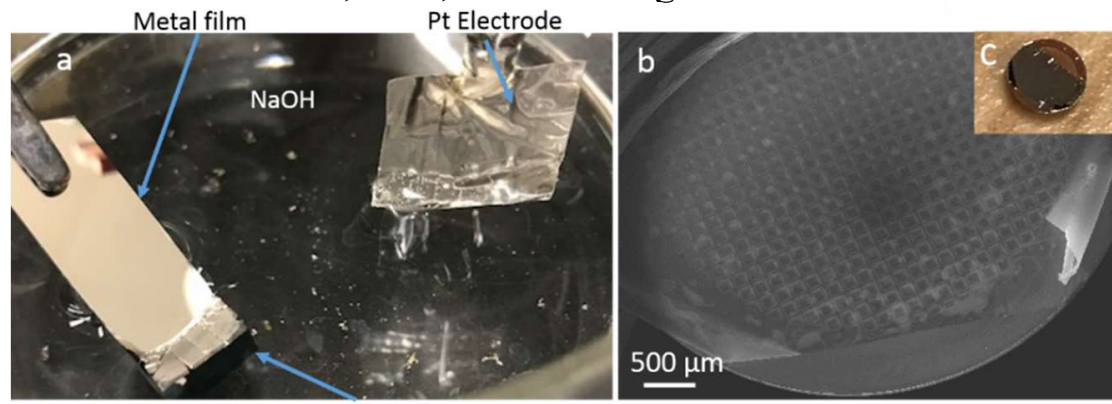

$\mathrm{SiO}$, support

Figure 1. a) Image of electrochemical transfer mechanism displaying the metal thin film delaminating from the $\mathrm{SiO}_{2}$ support wafer in $\mathrm{NaOH}$ electrolyte. b) SEM image of metal thin film on TEM support grid with c) optical image inset.
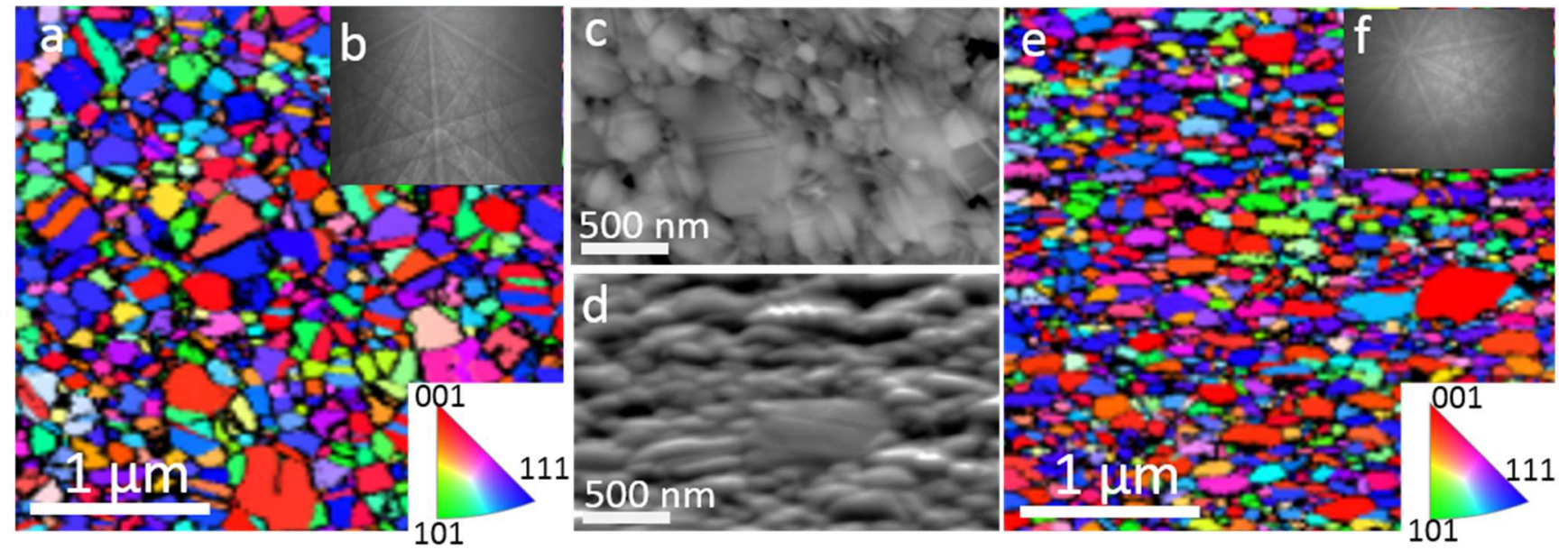

Figure 2. a) Unprocessed TKD IPF $Z$ map with b) inset sample Kikuchi pattern and c) FSD image of transferred metal thin film d) FSD image and e) Unprocessed IPF Z EBSD map with f) inset sample Kikuchi pattern of metal thin film on $\mathrm{SiO}_{2}$ support before transfer.
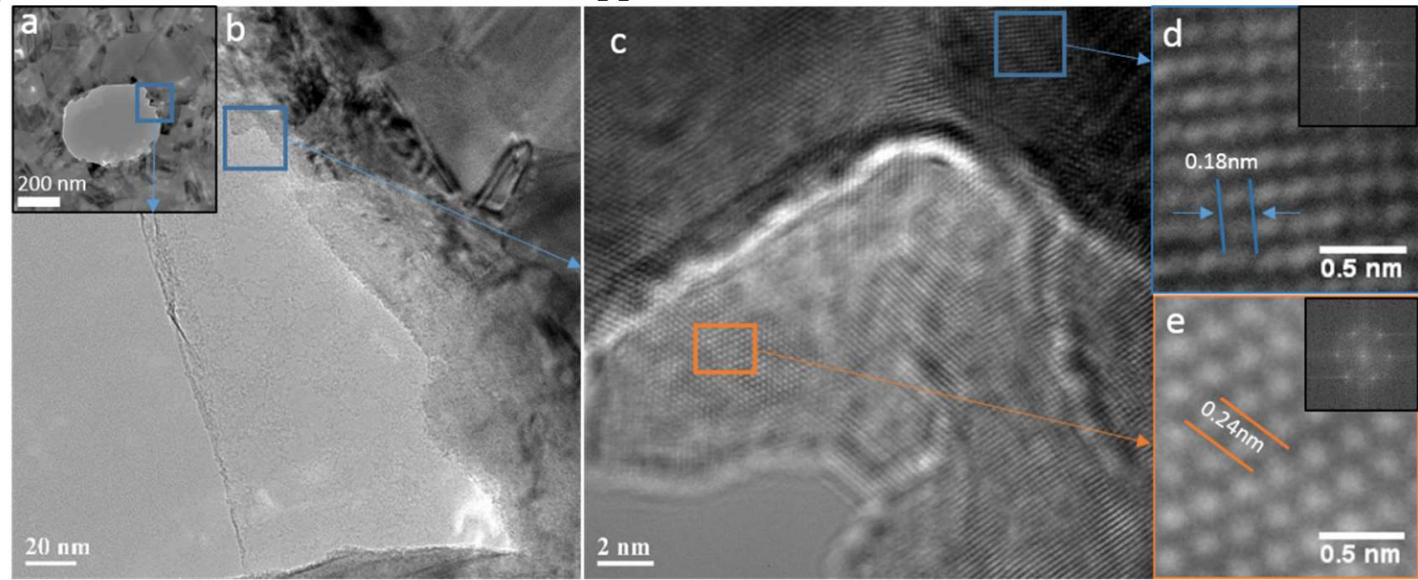

Figure 3. a) Bright field TEM image of hole in transferred metal thin film. b) Magnified section of the blue box in a) with both metal and graphene visible. c) Magnified image of the blue box in b) with both d) Ni lattice fringes and e) graphene lattice fringes visible. 